\title{
SUPPLEMENTAL ARTICLE TO
}

\section{Properties and numerical evaluation of the Rosenblatt distribution}

\section{Mark S. Veillette and Murad S. Taqqu}

In this supplemental article we provide additional details, tables and plots about the numerical methods used to compute distributional quantities related to the Rosenblatt distribution.

We often refer to the main article but some short portions are repeated for the sake of clarity. In Sections A and B, we provide details about the computation of the integral operator $\mathcal{K}_{D}: L^{2}(0,1) \rightarrow L^{2}(0,1), 0<D<1 / 2$, defined as

$$
\left(\mathcal{K}_{D} f\right)(x)=\int_{0}^{1}|x-u|^{-D} f(u) d u
$$

in (13). We compute the moments and cumulants in Section C, the eigenvalues in Section $\mathrm{D}$ and the CDF in Section E.

\section{Appendix A: Approximation of the integral operator $\mathcal{K}_{D}$}

The integral operator $\mathcal{K}_{D}$ plays an important role in each of these quantities, and so we will begin by presenting a method for approximating this integral operator with a matrix.

Fix $J>0$ and choose nodes $0=x_{0}<x_{1}<\cdots<x_{J}=1$. These nodes can be chosen as evenly spaced, $x_{j}=j / J$, however we noticed slightly better results with non-evenly space nodes that are more dense around 0 and 1 , for example nodes chosen as

$$
x_{j}=\left\{\begin{array}{l}
8\left(\frac{j}{J}\right)^{4}, \quad j \leq J / 2 \\
1-8\left(1-\left(\frac{j}{J}\right)\right)^{4}, \quad j>J / 2
\end{array}\right.
$$

Using these nodes, we approximate a continuous function $f \in L^{2}(0,1)$ using a linear interpolation of $f$ on the nodes $x_{n}$. For such $f$, let $\mathbf{f}=\left(f\left(x_{0}\right), \ldots, f\left(x_{J}\right)\right)$ and define

$$
f_{J}(x)=f\left(x_{j-1}\right) \frac{x-x_{j}}{x_{j-1}-x_{j}}+f\left(x_{j}\right) \frac{x-x_{j-1}}{x_{j}-x_{j-1}}, \quad \text { for } x \in\left[x_{j-1}, x_{j}\right]
$$


Properties and numerical evaluation of the Rosenblatt distribution

Then, for any $i=0,1, \ldots, J$,

$$
\begin{aligned}
\left(\mathcal{K}_{D} f_{J}\right)\left(x_{i}\right) & =\sum_{j=1}^{J} \int_{x_{j-1}}^{x_{j}}\left(f\left(x_{j-1}\right) \frac{u-x_{j}}{x_{j-1}-x_{j}}+f\left(x_{j}\right) \frac{u-x_{j-1}}{x_{j}-x_{j-1}}\right)\left|x_{i}-u\right|^{-D} d u \\
& =c_{i, 0} f\left(x_{0}\right)+\sum_{j=1}^{J-1}\left(c_{i, j}+d_{i, j}\right) f\left(x_{j}\right)+d_{i, J} f\left(x_{J}\right) \\
& =\left(\mathbf{K}_{D} \mathbf{f}\right)_{i},
\end{aligned}
$$

where $\mathbf{K}_{D}$ is an $(J+1) \times(J+1)$ matrix given by $\mathbf{K}_{D}=\mathbf{c}+\mathbf{d}$, where the matrices $\mathbf{c}=\left(c_{i, j}\right)_{i, j=0}^{J}$ and $\mathbf{d}=\left(d_{i, j}\right)_{i, j=0}^{J}$ are given by $c_{i, J}=d_{i, 0}=0$ and for $i=0,1, \ldots, J$ are

$$
\begin{aligned}
c_{i, j}=\frac{1}{x_{j}-x_{j+1}} \int_{x_{j}}^{x_{j+1}}\left(u-x_{j+1}\right)\left|x_{i}-u\right|^{-D} d u, \quad j=0,1, \ldots, J-1 \\
d_{i, j}=\frac{1}{x_{j}-x_{j-1}} \int_{x_{j-1}}^{x_{j}}\left(u-x_{j-1}\right)\left|x_{i}-u\right|^{-D} d u, \quad j=1,2, \ldots, J
\end{aligned}
$$

These integrals are computed in Section B and are given by

$$
\begin{aligned}
& c_{i, j}=\left\{\begin{array}{l}
\frac{\left(x_{i}-x_{j}\right)^{1-D}}{(1-D)}+\frac{\left(x_{i}-x_{j+1}\right)^{2-D}-\left(x_{i}-x_{j}\right)^{2-D}}{(1-D)(2-D)\left(x_{j+1}-x_{j}\right)}, \quad 0 \leq j<i \\
\frac{\left(x_{j+1}-x_{i}\right)^{2-D}-\left(x_{j}-x_{i}\right)^{2-D}}{(1-D)(2-D)\left(x_{j+1}-x_{j}\right)}-\frac{\left(x_{j}-x_{i}\right)^{1-D}}{(1-D)} \quad i \leq j<J
\end{array}\right. \\
& d_{i, j}= \begin{cases}\frac{\left(x_{i}-x_{j-1}\right)^{2-D}-\left(x_{i}-x_{j}\right)^{2-D}}{(1-D)(2-D)\left(x_{j}-x_{j-1}\right)}-\frac{\left(x_{i}-x_{j}\right)^{2-D}}{(1-D)} & 0<j \leq i \\
\frac{\left(x_{j}-x_{i}\right)^{1-D}}{(1-D)}-\frac{\left(x_{j}-x_{i}\right)^{2-D}-\left(x_{j-1}-x_{i}\right)^{2-D}}{(1-D)(2-D)\left(x_{j}-x_{j-1}\right)} & i<j \leq J\end{cases}
\end{aligned}
$$
$\mathcal{K}_{D}$.

Below, we use this matrix $\mathbf{K}_{D}$ to approximate the cumulants and the eigenvalues of

\section{Appendix B: Computation of $\mathrm{K}_{D}$}

We give here details of the computation of the entries $c_{i, j}$ and $d_{i, j}$ in the matrix $\mathbf{K}_{D}$ which are given in (80) and (81). These are given for each $i=0,1, \ldots, J$ by $c_{i, J}=d_{i, 0}=0$ and

$$
\begin{aligned}
& c_{i, j}=\frac{1}{x_{j}-x_{j+1}} \int_{x_{j}}^{x_{j+1}}\left(u-x_{j+1}\right)\left|x_{i}-u\right|^{-D} d u, j=0,1, \ldots, J-1 \\
& d_{i, j}=\frac{1}{x_{j}-x_{j-1}} \int_{x_{j-1}}^{x_{j}}\left(u-x_{j-1}\right)\left|x_{i}-u\right|^{-D} d u, \quad j=1,2, \ldots, J
\end{aligned}
$$


We start with the computation of $c_{i, j}$. If $j<i$ then $x_{j+1} \leq x_{i}$ and we can remove the absolute values in the integral and integrate by parts

$$
\begin{aligned}
\int_{x_{j}}^{x_{j+1}}\left(u-x_{j+1}\right)\left|x_{i}-u\right|^{-D} d u & =\int_{x_{j}}^{x_{j+1}}\left(u-x_{j+1}\right)\left(x_{i}-u\right)^{-D} d u \\
& =\frac{\left(x_{j}-x_{j+1}\right)\left(x_{i}-x_{j}\right)^{1-D}}{(1-D)}+\frac{1}{(1-D)} \int_{x_{j}}^{x_{j+1}}\left(x_{i}-u\right)^{1-D} d u \\
& =\frac{\left(x_{j}-x_{j+1}\right)\left(x_{i}-x_{j}\right)^{1-D}}{(1-D)}-\frac{\left(x_{i}-x_{j+1}\right)^{2-D}-\left(x_{i}-x_{j}\right)^{2-D}}{(1-D)(2-D)}
\end{aligned}
$$

Then, dividing through by $\left(x_{j}-x_{j+1}\right)$ gives the $0 \leq j<i$ case in (80).

If $j \geq i$ then $x_{j} \geq x_{i}$ and so a similar calculation gives

$$
\begin{aligned}
\int_{x_{j}}^{x_{j+1}}\left(u-x_{j+1}\right)\left|x_{i}-u\right|^{-D} d u & =\int_{x_{j}}^{x_{j+1}}\left(u-x_{j+1}\right)\left(u-x_{i}\right)^{-D} d u \\
& =-\frac{\left(x_{j}-x_{j+1}\right)\left(x_{j}-x_{i}\right)^{1-D}}{(1-D)}-\frac{1}{1-D} \int_{x_{j}}^{x_{j+1}}\left(u-x_{i}\right)^{1-D} d u \\
& =-\frac{\left(x_{j}-x_{j+1}\right)\left(x_{j}-x_{i}\right)^{1-D}}{(1-D)}-\frac{\left(x_{j+1}-x_{i}\right)^{2-D}-\left(x_{j}-x_{i}\right)^{2-D}}{(1-D)(2-D)}
\end{aligned}
$$

and then dividing by $\left(x_{j}-x_{j+1}\right)$ gives the $i \leq j<J$ case in (80).

The computation for $d_{i, j}$ is similar. If $j \leq i$ then $x_{j} \leq x_{i}$ and

$$
\begin{aligned}
\int_{x_{j-1}}^{x_{j}}\left(u-x_{j-1}\right)\left|x_{i}-u\right|^{-D} d u & =\int_{x_{j-1}}^{x_{j}}\left(u-x_{j-1}\right)\left(x_{i}-u\right)^{-D} d u \\
& =-\frac{\left(x_{j}-x_{j-1}\right)\left(x_{i}-x_{j}\right)^{1-D}}{(1-D)}+\frac{1}{1-D} \int_{x_{j-1}}^{x_{j}}\left(x_{i}-u\right)^{1-D} d u \\
& =-\frac{\left(x_{j}-x_{j-1}\right)\left(x_{i}-x_{j}\right)^{1-D}}{(1-D)}-\frac{\left(x_{i}-x_{j}\right)^{2-D}-\left(x_{i}-x_{j-1}\right)^{2-D}}{(1-D)(2-D)} .
\end{aligned}
$$

Dividing by $\left(x_{j}-x_{j-1}\right)$ confirms the $0<j<i$ case in (81). Finally, if $j>i$, then $x_{i} \leq x_{j-1}$ and

$$
\begin{aligned}
\int_{x_{j-1}}^{x_{j}}\left(u-x_{j-1}\right)\left|x_{i}-u\right|^{-D} d u & =\int_{x_{j-1}}^{x_{j}}\left(u-x_{j-1}\right)\left(u-x_{i}\right)^{-D} d u \\
& =\frac{\left(x_{j}-x_{j-1}\right)\left(x_{j}-x_{i}\right)^{1-D}}{(1-D)}-\frac{1}{(1-D)} \int_{x_{j-1}}^{x_{j}}\left(u-x_{i}\right)^{1-D} d u \\
& =\frac{\left(x_{j}-x_{j-1}\right)\left(x_{j}-x_{i}\right)^{1-D}}{(1-D)}-\frac{\left(x_{j}-x_{i}\right)^{2-D}-\left(x_{j-1}-x_{i}\right)^{2-D}}{(1-D)(2-D)}
\end{aligned}
$$

Dividing by $x_{j}-x_{j-1}$ confirms the $i<j \leq J$ case in (81). 


\begin{tabular}{|l|l|l|l|l|l|l|}
\hline$D$ & $\kappa_{3}$ & $\kappa_{4}$ & $\kappa_{5}$ & $\kappa_{6}$ & $\kappa_{7}$ & $\kappa_{8}$ \\
\hline 0 & 2.828 & 12 & 67.88 & 480.0 & 4073 & 40320.0 \\
0.05 & 2.815 & 11.92 & 67.33 & 475.3 & 4027 & 39797.5 \\
0.10 & 2.770 & 11.66 & 65.46 & 459.5 & 3871 & 38043.7 \\
0.15 & 2.684 & 11.15 & 61.92 & 429.9 & 3581 & 34805.9 \\
0.20 & 2.548 & 10.35 & 56.37 & 384.0 & 3139 & 29938.5 \\
0.25 & 2.348 & 9.192 & 48.51 & 320.6 & 2543 & 23537.4 \\
0.30 & 2.067 & 7.632 & 38.32 & 241.4 & 1826 & 16117.0 \\
0.35 & 1.686 & 5.665 & 26.24 & 153.0 & 1072 & 8767.1 \\
0.40 & 1.183 & 3.392 & 13.68 & 69.76 & 428.4 & 3072.7 \\
0.425 & 0.8844 & 2.235 & 8.059 & 36.89 & 203.6 & 1312.2 \\
0.45 & 0.5603 & 1.173 & 3.563 & 13.81 & 64.60 & 353.3 \\
0.475 & 0.2338 & 0.349 & 0.7735 & 2.199 & 7.560 & 30.40 \\
0.5 & 0 & 0 & 0 & 0 & 0 & 0 \\
\hline
\end{tabular}

Table 4. Cumulants of the Rosenblatt distribution for various $D$ which are given by (10). They are standardized, so $\kappa_{1}=0$ and $\kappa_{2}=1$. To obtain $\kappa_{3}$, we computed $c_{3}$ using (12) and to get $\kappa_{4}$, we computed $G_{2, D}$ using (18) and $c_{4}=\left(G_{2, D}, G_{2, D}\right)$ from (15). The cumulants of higher orders are evaluated using (83).

\section{Appendix C: Computation of the cumulants and moments}

In Section 2, we showed in Proposition 2.1 that the cumulants (and hence moments) of $Z_{D}$ can be expressed in terms of the functions $G_{k, D}$ defined recursively in (14). $G_{2, D}$ can be computed in terms of the beta function and the Gauss hypergeometric function, see (18). Thus, $c_{4}$ and hence $\kappa_{4}$ can be computed using numerical integration. A closed form for $G_{k, D}$ could not be found, and so a numerical method is required to approximate these functions.

In order to compute $G_{k, D}$ for $k>2$, define $\mathbf{G}_{2, D}=\left(G_{2, D}\left(x_{0}\right), \ldots, G_{2, D}\left(k_{J}\right)\right)$ which can be computed using (18). Then, the vector $\mathbf{G}_{k, D}=\left(G_{k, D}\left(x_{0}\right), \ldots, G_{k, D}\left(x_{J}\right)\right)$ for $k>2$ can be approximated by

$$
\mathbf{K}_{D}^{k-1} \mathbf{G}_{2, D}
$$

By Proposition 2.1, if $\mu+\nu=k$ we have $c_{k}=\left(G_{\mu, D}, G_{\nu, D}\right)$. Thus $c_{k}$ can be approximated using the trapezoid rule:

$$
c_{k} \approx \sum_{i=1}^{J} \frac{1}{2}\left(x_{i}-x_{i-1}\right)\left[\left(\mathbf{K}_{D}^{\mu-1} \mathbf{G}_{2, D}\right)_{i-1}\left(\mathbf{K}_{D}^{\nu-1} \mathbf{G}_{2, D}\right)_{i-1}+\left(\mathbf{K}_{D}^{\mu-1} \mathbf{G}_{2, D}\right)_{i}\left(\mathbf{K}_{D}^{\nu-1} \mathbf{G}_{2, D}\right)_{i}\right] .
$$

Having obtained the $c_{k}$ 's we get the cumulants using (10), and then we get the moments by applying (11). We've tabulated the first 8 moments and cumulants of the Rosenblatt distribution for various $D$ in Tables 4 and 5 . 


\begin{tabular}{|l|l|l|l|l|l|l|}
\hline$D$ & $\mu_{3}$ & $\mu_{4}$ & $\mu_{5}$ & $\mu_{6}$ & $\mu_{7}$ & $\mu_{8}$ \\
\hline 0 & 2.828 & 15 & 96.17 & 755.0 & 6983 & 74417.0 \\
0.05 & 2.815 & 14.92 & 95.48 & 748.4 & 6911 & 73522.3 \\
0.10 & 2.770 & 14.66 & 93.16 & 726.1 & 6666 & 70519.9 \\
0.15 & 2.684 & 14.15 & 88.76 & 684.2 & 6211 & 64967.3 \\
0.20 & 2.548 & 13.35 & 81.85 & 619.1 & 5513 & 56577.8 \\
0.25 & 2.348 & 12.19 & 71.99 & 528.6 & 4564 & 45428.9 \\
0.30 & 2.067 & 10.63 & 58.99 & 413.6 & 3400 & 32254.0 \\
0.35 & 1.686 & 8.665 & 43.09 & 281.3 & 2134 & 18740.0 \\
0.40 & 1.183 & 6.392 & 25.51 & 149.6 & 980.3 & 7544.0 \\
0.425 & 0.8844 & 5.235 & 16.90 & 93.24 & 534.9 & 3712.6 \\
0.45 & 0.5603 & 4.173 & 9.166 & 49.54 & 221.3 & 1339.0 \\
0.475 & 0.2338 & 3.349 & 3.112 & 22.98 & 51.21 & 300.0 \\
0.5 & 0 & 3 & 0 & 15 & 0 & 105 \\
\hline
\end{tabular}

Table 5. Moments of the Rosenblatt distribution for various $D$. They are standardized, so $\mu_{1}=0$ and $\mu_{2}=1$. These are obtained from the cumulants by using (11).

\section{Appendix D: Computation of the eigenvalues}

There exists extensive literature regarding the problem of approximating eigenvalues of integral operators like $\mathcal{K}_{D}$, see for instance [5], [23], [2], or [10].

Many, if not all, of these methods boil down to approximating $\mathcal{K}_{D}$ with a finitedimensional linear operator defined on a subspace of $L^{2}(0,1)$, with $J$ indicating the level of approximation. We've already computed such a linear approximation, $\mathbf{K}_{D}$, to $\mathcal{K}_{D}$ above, where this subspace corresponds to all piecewise linear function of the nodes $x_{0}<x_{1}<\ldots x_{J}$. By taking the eigenvalues of the matrix $\mathbf{K}_{D}$, we can approximate the $M$ largest eigenvalues of $\mathcal{K}_{D}$ where $M \ll J$.

In order to test that the approximate eigenvalues we are obtaining are accurate, we have three methods:

1. Check for numerical convergence, i.e. for $J$ large, does increasing $J$ lead to a negligible change in the approximations of $\lambda_{n}$. Thus, to approximate $\lambda_{n}$, we increased $J$ until sufficient convergence was met. By "sufficient convergence", we mean that $J$ is increased by multiples of 200 until the values of the $\lambda_{n}$ 's no longer changed in the 4 significant decimal digit. Table 6 gives the results of this method applied to the first 30 values of $\lambda_{n}$ 's.

2. Compare $\lambda_{n}$ with the asymptotic formula given by Theorem 3.2 for $n$ large. The first $30 \lambda_{n}$ are approximated and plotted on a log scale in Figure 2 and compared to the asymptotic formula in Theorem 3.2. For large $n$, our approximations appear to be in agreement with the asymptotic formula as $n \rightarrow \infty$. In fact, the asymptotic formula for the $\lambda_{n}$ is a good approximation even for moderate values of $n$ (about $n \geq 20$ for $D=0.1,0.2,0.3,0.4)$. 


\begin{tabular}{|l|l|l|l|l|}
\hline$n$ & $D=0.1$ & $D=0.2$ & $D=0.3$ & $D=0.4$ \\
\hline 1 & 0.70200 & 0.68130 & 0.63050 & 0.51250 \\
2 & 0.05648 & 0.11260 & 0.16040 & 0.17840 \\
3 & 0.03477 & 0.07341 & 0.11070 & 0.13010 \\
4 & 0.02453 & 0.05411 & 0.08512 & 0.10420 \\
5 & 0.01947 & 0.04409 & 0.07119 & 0.08948 \\
6 & 0.01600 & 0.03710 & 0.06129 & 0.07879 \\
7 & 0.01374 & 0.03240 & 0.05446 & 0.07121 \\
8 & 0.01198 & 0.02872 & 0.04903 & 0.06512 \\
9 & 0.01069 & 0.02596 & 0.04489 & 0.06037 \\
10 & 0.009629 & 0.02366 & 0.04140 & 0.05635 \\
11 & 0.008792 & 0.02182 & 0.03858 & 0.05304 \\
12 & 0.008074 & 0.02023 & 0.03612 & 0.05014 \\
13 & 0.007485 & 0.01892 & 0.03405 & 0.04767 \\
14 & 0.006966 & 0.01775 & 0.03221 & 0.04546 \\
15 & 0.006528 & 0.01675 & 0.03063 & 0.04353 \\
16 & 0.006135 & 0.01586 & 0.02919 & 0.04178 \\
17 & 0.005796 & 0.01507 & 0.02792 & 0.04022 \\
18 & 0.005487 & 0.01436 & 0.02677 & 0.03879 \\
19 & 0.005216 & 0.01373 & 0.02573 & 0.03750 \\
20 & 0.004967 & 0.01314 & 0.02477 & 0.03630 \\
21 & 0.004746 & 0.01262 & 0.02391 & 0.03521 \\
22 & 0.004540 & 0.01214 & 0.02310 & 0.03419 \\
23 & 0.004355 & 0.01169 & 0.02236 & 0.03325 \\
24 & 0.004183 & 0.01128 & 0.02167 & 0.03236 \\
25 & 0.004026 & 0.01090 & 0.02103 & 0.03155 \\
26 & 0.003879 & 0.01055 & 0.02043 & 0.03077 \\
27 & 0.003744 & 0.01022 & 0.01988 & 0.03005 \\
28 & 0.003617 & 0.009914 & 0.01935 & 0.02937 \\
29 & 0.003500 & 0.009627 & 0.01886 & 0.02873 \\
30 & 0.003389 & 0.009355 & 0.01839 & 0.02812 \\
\hline
\end{tabular}

Table 6. First 30 eigenvalues of $\mathcal{K}_{D}$ for various $D$. Note that for $D=0$, we have $\lambda_{1}=1$ and $\lambda_{n}=0$ for $n \geq 2$. As $D \rightarrow 1 / 2, \lambda_{n} \rightarrow 0$.

3. The cumulants $\kappa_{k}$ of $Z_{D}$ are given in terms of the eigenvalues as

$$
\kappa_{k}=2^{k-1}(k-1) ! \sum_{n=1}^{\infty} \lambda_{n}^{k}=2^{k-1}(k-1) !\left[\sum_{n=1}^{M-1} \lambda_{n}^{k}+\sum_{n=M}^{\infty} \lambda_{n}^{k}\right] \text {, }
$$

Since $\kappa_{2}=1$, and $\kappa_{3}, \kappa_{4}$ can be computed exactly (see the caption of Table 4 ), we can compute the absolute error made by approximating the cumulants $\kappa_{2}, \kappa_{3}, \kappa_{4}$ by using (84). In order to compute the sums on the right hand side of (84), we used the first $M=50$ values of $\lambda_{n}$ using the matrix $\mathbf{K}_{D}$, and for $n>M$, the asymptotic formula given in Theorem 3.2. The results of this test are given in Table 7. Because we are using the asymptotic formula for $n$ large, we expect some error, but as it turns out, the absolute errors are very small compared to the size of $\kappa_{k}, k=2,3,4$. 


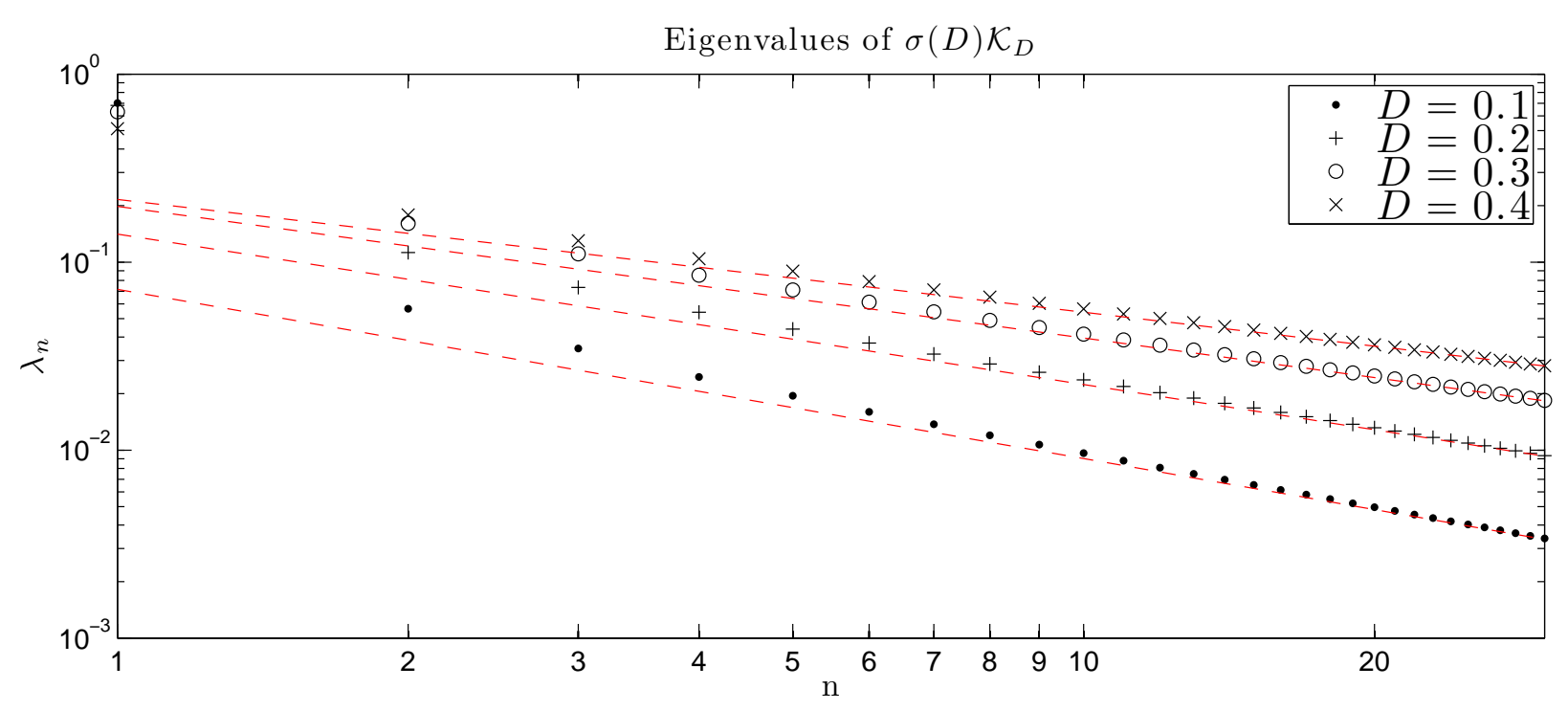

Figure 2. Approximations of the first 30 eigenvalues of $\sigma(D) \mathcal{K}_{D}$ for $D=0.1,0.2,0.3$ and 0.4 on a $\log$-log plot. The dashed line represent the power law $C(D) n^{D-1}$ given by (29). We can see how quickly this asymptotic formula gives an accurate approximation.

\begin{tabular}{|l|l|l|l|}
\hline$D$ & Error in $\kappa_{2}$ & Error in $\kappa_{3}$ & Error in $\kappa_{4}$ \\
\hline 0.1 & $0.1620 \times 10^{-4}$ & $0.0259 \times 10^{-4}$ & $0.1243 \times 10^{-4}$ \\
0.2 & $0.1198 \times 10^{-3}$ & $0.0106 \times 10^{-3}$ & $0.0232 \times 10^{-3}$ \\
0.3 & $0.4621 \times 10^{-3}$ & $0.0487 \times 10^{-3}$ & $0.0340 \times 10^{-3}$ \\
0.4 & $0.7805 \times 10^{-3}$ & $0.0908 \times 10^{-3}$ & $0.0257 \times 10^{-3}$ \\
\hline
\end{tabular}

Table 7. Errors in approximating the cumulants $\kappa_{k}$ using the eigenvalues as obtained in Section D with $J=1500$. These numbers are small which suggests that the method is closely approximating the eigenvalues.

\section{Appendix E: Computation of the CDF}

This section ties together the results of the previous sections to devise a technique for computing the CDF and PDF of the Rosenblatt distribution. Computing the distribution of $Z_{D}$ requires three steps:

1. Approximate the eigenvalues $\lambda_{n}$ for $n=1,2, \ldots, M$ for some $M>0$.

2. Compute separately the $\mathrm{CDF}$ of $X_{M}$ and the PDF of $Y_{M}$ in the decomposition (65).

For $X_{M}$, methods exits already for accurately computing the CDF of a finite sum 
Properties and numerical evaluation of the Rosenblatt distribution

of chi-squared distributions, see for instance methods based on Laplace transform inversion, [34], [9], or Fourier transform inversion, [1].

For $Y_{M}$, use an Edgeworth expansion of order $N \geq 2$ which is found in Theorem 5.2. To compute $\sigma_{M}$ in (66), take

$$
\sigma_{M}=\sqrt{1-2 \sum_{n=1}^{M-1} \lambda_{n}^{2}}
$$

The Edgeworth expansion in Theorem 5.2 also involves the cumulants $\kappa_{k, M}, k \geq 1$ of $Y_{M}$. If $M$ is sufficiently large, $\kappa_{k, M}$ can be approximated using the asymptotic formula given in Theorem 3.2:

$$
\begin{aligned}
\kappa_{k, M} & =\sigma_{M}^{-k}\left(2^{k-1}(k-1) ! \sum_{n=M}^{\infty} \lambda_{n}^{k}\right) \\
& \approx \sigma_{M}^{-k}\left(2^{k-1}(k-1) ! \sum_{n=M}^{\infty} C(D)^{k} n^{k(D-1)}\right) \\
& =2^{k-1}(k-1) ! \sigma_{M}^{-k} C(D)^{k} \zeta(k(1-D), M)
\end{aligned}
$$

where $\zeta(s, M)=\sum_{n=M}^{\infty} n^{-s}$ denotes the Hurwitz Zeta function ([16], chapter 64). Using (85) introduces a small amount of error since we are approximating $\lambda_{n}$ for $n$ large, however we will see below this is negligible for $M$ large. As an alternative, $\kappa_{k, M}$ can also be computed using the methods in Section C, and then $\kappa_{k, M}$ can be computed using the first $M-1 \lambda_{n}$ 's:

$$
\kappa_{k, M}=\sigma_{M}^{-k}\left[\kappa_{k}-2^{k-1}(k-1) ! \sum_{n=1}^{M-1} \lambda_{n}^{k}\right],
$$

however this is numerically unstable for $M$ large, since $\sigma_{M}$ is small and $\kappa_{k}$ is very close to $2^{k-1}(k-1) ! \sum_{n=1}^{M-1} \lambda_{n}^{k}$. For this reason, we used (77) to compute $\kappa_{k, M}$.

3. Finally, the CDF of $Z_{D}$ is given by a convolution

$$
F_{Z_{D}}(x)=\int_{-\infty}^{\infty} F_{X_{M}}(x-y) f_{Y_{M}}(y) d y
$$

where $F_{X_{M}}$ is the CDF of $X_{M}$ and $f_{Y_{M}}$ is the PDF of $Y_{M}$. We compute this convolution in MATLAB using standard numerical integration techniques.

The choice of $M$ (number of chi-squared distributions) and $N$ (order of Edgeworth expansion) was determined by increasing both until the value of the CDF changed by less than $10^{-5}$. In Table 8 , we show the effects of increasing $M$ and $N$ for the case of $D=0.3$ and $x=0$. Observe that for fixed $M$, the values of the CDF converge rapidly as $N$ increases. Nevertheless, if $M$ is small $(M \leq 10)$ the approximation will have a slight 


\begin{tabular}{|l|l|l|l|l|}
\hline $\mathrm{N}$ & $M=10$ & $M=20$ & $M=30$ & $M=50$ \\
\hline 2 & 0.616883 & 0.616909 & 0.616909 & 0.616907 \\
3 & 0.616817 & 0.616878 & 0.616890 & 0.616896 \\
4 & 0.616885 & 0.616898 & 0.616899 & 0.616899 \\
5 & 0.616895 & 0.616900 & 0.616900 & 0.616900 \\
6 & 0.616895 & 0.616900 & 0.616900 & 0.616900 \\
\hline
\end{tabular}

Table 8. Approximation of $\mathrm{P}\left[Z_{0.3} \leq 0\right]$ for various $M$ and $N$ values. This shows that one gets an excellent approximation with relatively small values of $M$ and $N$.

error since we are approximating the $\kappa_{k, M}$ using (77). Moreover, by choosing $N=2$ and $M \geq 10$, the only noticeable improvement by increasing $N$ happens in the fifth decimal place and beyond.

As a final verification, we plotted in Figure 3 the CDF of a chi-squared distribution with one degree of freedom, normalized to have mean 0 and variance 1 (i.e. $\mathrm{D}=0$ case), computed exactly at a succession of points with linear interpolation in between. Observe that if $C$ and $\widetilde{C}$ denote respectively a chi-squared random variable with one degree of freedom and its normalized version, then $\widetilde{C}=(C-1) / \sqrt{2}$ and $P[\widetilde{C} \leq c]=P[C \leq 1+c \sqrt{2}]$. In particular, the CDF of $\widetilde{C}$ is zero if $1+c \sqrt{2}<0$, that is, if $c<-1 / \sqrt{2}=-0.7071$, as observed in Figure 3. The figure also shows various values of the CDF computed with the software for the case $\mathrm{D}=0.01$. Since D is very small, the two CDFs should be very close, and indeed they are.

We've tabulated the values of the $\mathrm{CDF}$ of $Z_{D}$ for various $x$ and $D$ values in Table 9 . These are useful for obtaining percentiles and confidence intervals. To obtain these values, we fixed $N=6$, and increased $M$ in increments of 10 until the approximation of the CDF changed by less than $10^{-5}$. This level of convergence was achieved for all values of $x$ and $D$ considered.

\section{Appendix F: Guide to Software}

Software written in MATLAB is available which implements the methods described in the previous sections. The following functions are available:

RosenblattC

RosenblattEigs

RosenblattCDF

RosenblattPDF
Computes the cumulants and moments of the Rosenblatt distribution Computes the eigenvalues of the operator $\sigma(D) \mathcal{K}_{D}$. Computes the CDF of the Rosenblatt distribution Computes the PDF of the Rosenblatt distribution

Below, we will describe the use of each function and provide examples. 


\begin{tabular}{|c|c|c|c|c|c|c|c|c|c|c|c|}
\hline$x$ & $D=0.1$ & $D=0.2$ & $D=0.3$ & $\bar{D}=0.4$ & $D=0.45$ & $x$ & $D=0.1$ & $D=0.2$ & $D=0.3$ & $D=0.4$ & $D=0.45$ \\
\hline-2.5 & 0.0000 & 0.0000 & 0.0000 & 0.0002 & 0.0020 & 1.0 & 0.8797 & 0.8790 & 0.8755 & 0.8645 & 0.8532 \\
\hline-2.4 & 0.0000 & 0.0000 & 0.0000 & 0.0004 & 0.0030 & 1.1 & 0.8901 & 0.8899 & 0.8879 & 0.8805 & 0.8725 \\
\hline-2.3 & 0.0000 & 0.0000 & 0.0000 & 0.0007 & 0.0043 & 1.2 & 0.8996 & 0.8997 & 0.8989 & 0.8946 & 0.8897 \\
\hline-2.2 & 0.0000 & 0.0000 & 0.0000 & 0.0013 & 0.0062 & 1.3 & 0.9081 & 0.9086 & 0.9087 & 0.9071 & 0.9047 \\
\hline-2.1 & 0.0000 & 0.0000 & 0.0000 & 0.0022 & 0.0088 & 1.4 & 0.9159 & 0.9166 & 0.9174 & 0.9180 & 0.9179 \\
\hline-2 & 0.0000 & 0.0000 & 0.0000 & 0.0037 & 0.0122 & 1.5 & 0.9230 & 0.9238 & 0.9253 & 0.9276 & 0.9294 \\
\hline-1.9 & 0.0000 & 0.0000 & 0.0000 & 0.0059 & 0.0167 & 1.6 & 0.9294 & 0.9304 & 0.9323 & 0.9360 & 0.9393 \\
\hline-1.8 & 0.0000 & 0.0000 & 0.0001 & 0.0093 & 0.0225 & 1.7 & 0.9353 & 0.9364 & 0.9386 & 0.9434 & 0.9479 \\
\hline-1.7 & 0.0000 & 0.0000 & 0.0004 & 0.0142 & 0.0299 & 1.8 & 0.9406 & 0.9418 & 0.9443 & 0.9499 & 0.9554 \\
\hline-1.6 & 0.0000 & 0.0000 & 0.0011 & 0.0210 & 0.0392 & 1.9 & 0.9455 & 0.9467 & 0.9494 & 0.9556 & 0.9617 \\
\hline-1.5 & 0.0000 & 0.0000 & 0.0030 & 0.0303 & 0.0506 & 2.0 & 0.9499 & 0.9512 & 0.9541 & 0.9606 & 0.9672 \\
\hline-1.4 & 0.0000 & 0.0000 & 0.0070 & 0.0425 & 0.0645 & 2.1 & 0.9540 & 0.9553 & 0.9582 & 0.9650 & 0.9719 \\
\hline-1.3 & 0.0000 & 0.0000 & 0.0148 & 0.0582 & 0.0810 & 2.2 & 0.9577 & 0.9590 & 0.9620 & 0.9689 & 0.9758 \\
\hline-1.2 & 0.0000 & 0.0008 & 0.0282 & 0.0777 & 0.1003 & 2.3 & 0.9611 & 0.9624 & 0.9654 & 0.9724 & 0.9793 \\
\hline-1.1 & 0.0000 & 0.0052 & 0.0489 & 0.1014 & 0.1227 & 2.4 & 0.9643 & 0.9655 & 0.9685 & 0.9754 & 0.9822 \\
\hline-1 & 0.0000 & 0.0205 & 0.0785 & 0.1293 & 0.1481 & 2.5 & 0.9671 & 0.9684 & 0.9713 & 0.9781 & 0.9847 \\
\hline-0.9 & 0.0011 & 0.0554 & 0.1174 & 0.1616 & 0.1766 & 2.6 & 0.9697 & 0.9710 & 0.9739 & 0.9805 & 0.9868 \\
\hline-0.8 & 0.0344 & 0.1134 & 0.1649 & 0.1980 & 0.2081 & 2.7 & 0.9721 & 0.9733 & 0.9762 & 0.9826 & 0.9886 \\
\hline-0.7 & 0.1435 & 0.1893 & 0.2195 & 0.2380 & 0.2424 & 2.8 & 0.9744 & 0.9755 & 0.9783 & 0.9845 & 0.9902 \\
\hline-0.6 & 0.2754 & 0.2737 & 0.2789 & 0.2810 & 0.2792 & 2.9 & 0.9764 & 0.9775 & 0.9802 & 0.9861 & 0.9915 \\
\hline-0.5 & 0.3864 & 0.3575 & 0.3406 & 0.3264 & 0.3182 & 3.0 & 0.9782 & 0.9793 & 0.9819 & 0.9876 & 0.9926 \\
\hline-0.4 & 0.4726 & 0.4349 & 0.4022 & 0.3734 & 0.3588 & 3.1 & 0.9799 & 0.9810 & 0.9835 & 0.9889 & 0.9936 \\
\hline-0.3 & 0.5402 & 0.5035 & 0.4619 & 0.4211 & 0.4007 & 3.2 & 0.9815 & 0.9825 & 0.9849 & 0.9901 & 0.9944 \\
\hline-0.2 & 0.5947 & 0.5630 & 0.5180 & 0.4687 & 0.4433 & 3.3 & 0.983 & 0.9839 & 0.9862 & 0.9911 & 0.9952 \\
\hline-0.1 & 0.6400 & 0.6140 & 0.5698 & 0.5154 & 0.4861 & 3.4 & 0.9843 & 0.9852 & 0.9874 & 0.9921 & 0.9958 \\
\hline 0 & 0.6784 & 0.6577 & 0.6169 & 0.5605 & 0.5285 & 3.5 & 0.9855 & 0.9864 & 0.9885 & 0.9929 & 0.9963 \\
\hline 0.1 & 0.7115 & 0.6952 & 0.6591 & 0.6036 & 0.5700 & 3.6 & 0.9866 & 0.9875 & 0.9895 & 0.9936 & 0.9968 \\
\hline 0.2 & 0.7403 & 0.7277 & 0.6967 & 0.6441 & 0.6102 & 3.7 & 0.9877 & 0.9885 & 0.9904 & 0.9943 & 0.9972 \\
\hline 0.3 & 0.7655 & 0.7558 & 0.7300 & 0.6818 & 0.6488 & 3.8 & 0.9886 & 0.9894 & 0.9912 & 0.9949 & 0.9975 \\
\hline 0.4 & 0.7878 & 0.7805 & 0.7594 & 0.7165 & 0.6853 & 3.9 & 0.9895 & 0.9902 & 0.9919 & 0.9954 & 0.9978 \\
\hline 0.5 & 0.8077 & 0.8021 & 0.7853 & 0.7482 & 0.7196 & 4.0 & 0.9903 & 0.9910 & 0.9926 & 0.9959 & 0.9981 \\
\hline 0.6 & 0.8253 & 0.8213 & 0.8081 & 0.7769 & 0.7514 & 4.1 & 0.9910 & 0.9917 & 0.9933 & 0.9963 & 0.9983 \\
\hline 0.7 & 0.8412 & 0.8382 & 0.8282 & 0.8027 & 0.7807 & 4.2 & 0.9917 & 0.9924 & 0.9938 & 0.9967 & 0.9985 \\
\hline 0.8 & 0.8554 & 0.8534 & 0.8459 & 0.8258 & 0.8074 & 4.3 & 0.9924 & 0.9930 & 0.9943 & 0.9970 & 0.9986 \\
\hline 0.9 & 0.8682 & 0.8669 & 0.8616 & 0.8463 & 0.8315 & 4.4 & 0.9929 & 0.9935 & 0.9948 & 0.9973 & 0.9988 \\
\hline & & & & & & 4.5 & 0.9935 & 0.9940 & 0.9953 & 0.9976 & 0.9989 \\
\hline
\end{tabular}

Table 9. Values of the CDF $F(x)=\mathrm{P}\left[Z_{D} \leq x\right]$ for various values of $x$ and $D$ for the standardized Rosenblatt distribution. An entry of 0.0000 means the CDF takes a value less than $10^{-4}$. 


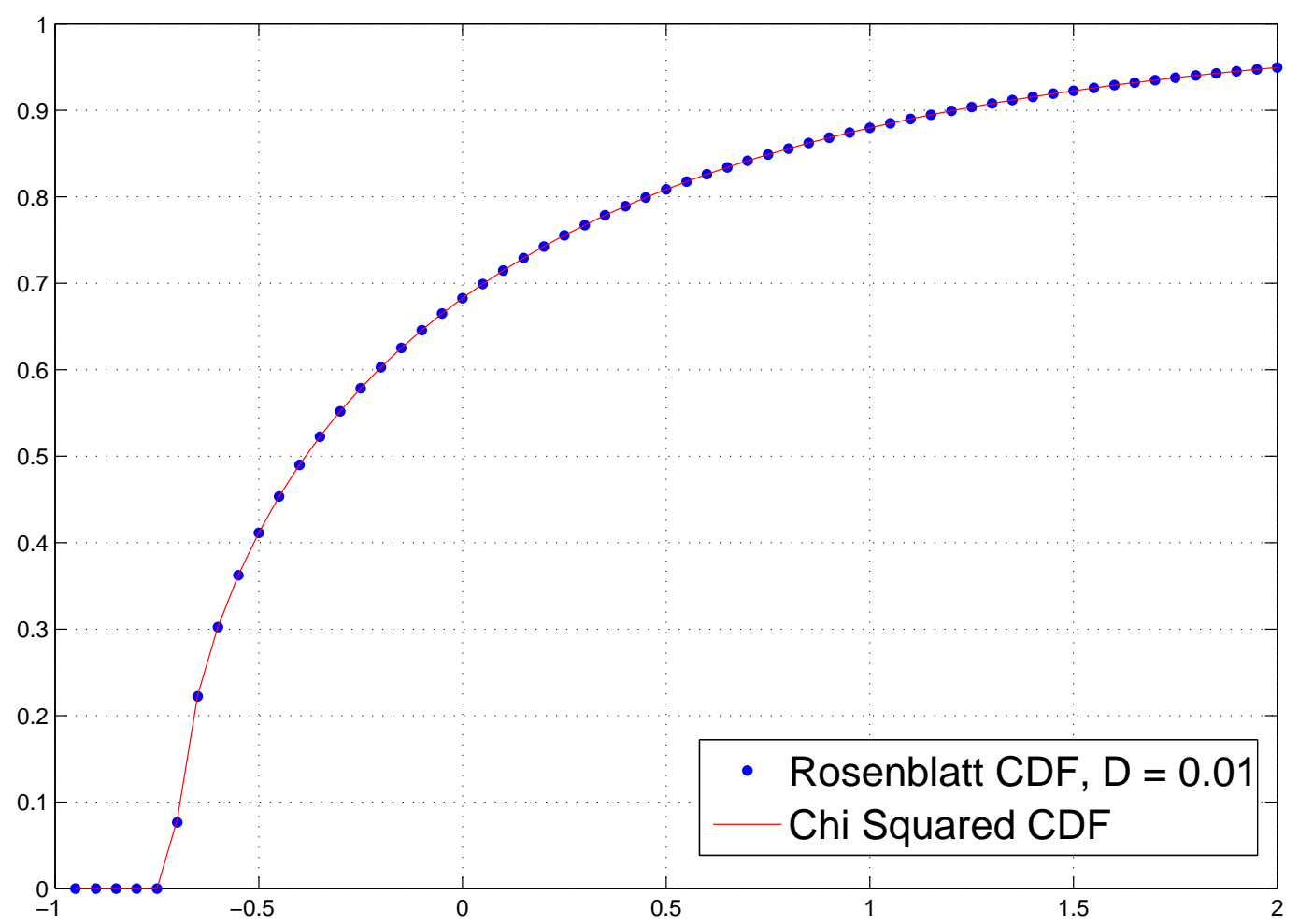

Figure 3. CDF of a chi-squared distribution with one degree of freedom, normalized to have mean 0 and variance 1 (i.e. $\mathrm{D}=0$ case), computed exactly at a succession of points with linear interpolation in between. Also various values of the $\mathrm{CDF}$ computed with the software for the case $\mathrm{D}=0.01$. Since $\mathrm{D}$ is very small, the two CDFs should be very close, and indeed they are.

\section{RosenblattC}

This function takes as input the following, in order:

$$
\begin{array}{ll}
D \in\left(0, \frac{1}{2}\right) & \text { Parameter of Rosenblatt distribution } \\
K \geq 2 & \text { Number of cumulants or moments to } \\
\text { 'cumulants' or 'moments' } & \text { Return cumulants or moments }
\end{array}
$$


For example, entering

$\gg$ RosenblattC $(0.3,8$, 'moments' $)$

will return the first 8 moments of $Z_{0.3}$ in a column vector.

\section{RosenblattEigs}

This function computed the eigenvalues of the operator $\sigma(D) \mathcal{K}_{D}$ using the the technique described in Section D. It takes as input the following, in order:

$$
\begin{array}{ll}
D \in\left(0, \frac{1}{2}\right) & \text { Parameter of Rosenblatt distribution } \\
M \geq 2 & \text { Number of eigenvalues to be computed } \\
J \gg M & \text { size of mesh used in computation }
\end{array}
$$

For example,

$\gg$ RosenblattEigs $(0.3,30,500)$

will return in a column vector the largest 30 eigenvalues of $\sigma(0.3) \mathcal{K}_{0.3}$ using a mesh of size 500 .

\section{RosenblattCDF \& RosenblattPDF}

These functions take as input the following in order to compute the CDF $F_{Z_{D}}(x)$ or the $\operatorname{PDF} f_{Z_{D}}(x)$ of the Rosenblatt distribution:

$$
\begin{array}{ll}
x \in \mathbb{R} & \text { Argument of PDF/CDF } \\
D \in\left(0, \frac{1}{2}\right) & \text { Parameter of Rosenblatt distribution }
\end{array}
$$

In addition, one can also provide the following two optional parameters. If these are not entered, the code will automatically choose them to ensure numerical convergence, namely by fixing $N=6$ and increasing $M$ until one sees a change of less than $10^{-5}$.

$$
\begin{array}{ll}
M \geq 2 & \text { Number of chi-square distributions to use in approximation } \\
N \geq 2 & \text { Order of Edgeworth expansion to use }
\end{array}
$$


For example,

$\gg$ RosenblattCDF ([-2 -10012$], 0.25)$

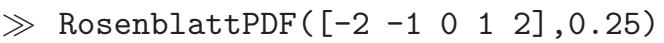

will return the values of the $\operatorname{CDF} F_{Z_{0.25}}(x)$ and the PDF $f_{Z_{0.25}}(x)$ for $x \in\{-2,-1,0,1,2\}$ in row vectors.

\section{References}

[1] J. Abate and W. Whitt. The Fourier-series method for inverting transforms of probability distributions. Queueing Systems Theory Appl., 10(1-2):5-87, 1992.

[2] M. Ahues, A. Largillier, and B. V. Limaye. Spectral computations for bounded operators, volume 18 of Applied Mathematics (Boca Raton). Chapman \& Hall/CRC, Boca Raton, FL, 2001.

[3] J. M. P. Albin. A note on Rosenblatt distributions. Statist. Probab. Lett., 40(1):8391, 1998.

[4] D. Applebaum. Lévy Processes and Stochastic Calculus. Cambridge University Press, Cambridge, UK, 2004.

[5] K. E. Atkinson. The numerical solutions of the eigenvalue problem for compact integral operators. Trans. Amer. Math. Soc., 129:458-465, 1967.

[6] K. Atkinson and W. Han. Theoretical Numerical Analysis. Springer, third edition, 2009.

[7] M. Š. Birman and M. Z. Solomjak. Estimates for the singular numbers of integral operators. Uspehi Mat. Nauk, 32(1(193)):17-84, 271, 1977.

[8] M. Braverman. On a class of Lévy processes. Statist. Probab. Lett., 75(3):179-189, 2005.

[9] A. Castaño-Martínez and F. López-Blázquez. Distribution of a sum of weighted noncentral chi-square variables. Test, 14(2):397-415, 2005.

[10] Z. Chen, G. Nelakanti, Y. Xu, and Y.Zhang. A fast collocation method for eigenproblems of weakly singular integral operators. J. Sci. Comput., 41(2):256-272, 2009.

[11] R. L. Dobrushin and P. Major. Non-central limit theorems for nonlinear functionals of Gaussian fields. Z. Wahrsch. Verw. Gebiete, 50(1):27-52, 1979.

[12] M. R. Dostanić. Spectral properties of the operator of Riesz potential type. Proc. Amer. Math. Soc., 126(8):2291-2297, 1998.

[13] I. S. Gradshteyn and I. M. Ryzhik. Table of Integrals, Series, and Products. Elsevier/Academic Press, Amsterdam, seventh edition, 2007. Translated from the Russian, Translation edited and with a preface by Alan Jeffrey and Daniel Zwillinger, With one CD-ROM (Windows, Macintosh and UNIX).

[14] M. Kac. Distribution of eigenvalues of certain integral operators. Michigan. Math. J., 3:141-148, 1955-1956. 
[15] P. Major. Multiple Wiener-Itô integrals, volume 849 of Lecture Notes in Mathematics. Springer, Berlin, 1981. With applications to limit theorems.

[16] K. Oldham, J. Myland, and J. Spanier. An Atlas of Functions. Springer, New York, second edition, 2009. With Equator, the atlas function calculator, With 1 CD-ROM (Windows).

[17] G. Peccati and M. S. Taqqu. Wiener Chaos: Moments, Cumulants and Diagrams. Bocconi Press and Springer Verlag, 2010. To appear.

[18] J. Pitman. Combinatorial stochastic processes, volume 1875 of Lecture Notes in Mathematics. Springer-Verlag, Berlin, 2006. Lectures from the 32nd Summer School on Probability Theory held in Saint-Flour, July 7-24, 2002, With a foreword by Jean Picard.

[19] M. Rosenblatt. Independence and dependence. In Proc. 4th Berkeley Sympos. Math. Statist. and Prob., Vol. II, pages 431-443. Univ. California Press, Berkeley, Calif., 1961.

[20] M. Rosenblatt. Some results on the asymptotic behavior of eigenvalues for a class of integral equations with translation kernels. J. Math. Mech., 12:619-628, 1963.

[21] K. Sato. Lévy processes and infinitely divisible distributions, volume 68 of Cambridge Studies in Advanced Mathematics. Cambridge University Press, Cambridge, 1999. Translated from the 1990 Japanese original, Revised by the author.

[22] I. G. Shevtsova. Sharpening the upper bound for the absolute constant in the BerryEsseen inequality. Teor. Veroyatn. Primen., 51(3):622-626, 2006.

[23] I. H. Sloan. Iterated Galerkin method for eigenvalue problems. SIAM J. Numer. Anal., 13(5):753-760, 1976.

[24] P. J. Smith. A recursive formulation of the old problem of obtaining moments from cumulants and vice versa. Amer. Statist., 49(2):217-218, 1995.

[25] A. Spence. On the convergence of the nyström method for the integral equation eigenvalue problem. Numerische Mathematik, 25:57-66, 1975.

[26] A. Spence. Error bounds and estimates for eigenvalues of integral equations. Numerische Mathematik, 29:133-146, 1978.

[27] E. Stade. Fourier Analysis. Pure and Applied Mathematics (New York). WileyInterscience [John Wiley \& Sons], Hoboken, NJ, 2005.

[28] F. W. Steutel and K. van Harn. Infinite divisibility of probability distributions on the real line, volume 259 of Monographs and Textbooks in Pure and Applied Mathematics. Marcel Dekker Inc., New York, 2004.

[29] M. S. Taqqu. Weak convergence to fractional Brownian motion and to the Rosenblatt process. Z. Wahrscheinlichkeitstheorie und Verw. Gebiete, 31:287-302, 1974/75.

[30] M. S. Taqqu. Convergence of integrated processes of arbitrary Hermite rank. $Z$. Wahrsch. Verw. Gebiete, 50(1):53-83, 1979.

[31] M. S. Taqqu. The Rosenblatt process. In Richard Davis, Keh-Shin Lii, and Dimitris Politis, editors, Selected Works of Murray Rosenblatt. Springer Verlag, New York., 2011.

[32] C. A. Tudor. Analysis of the Rosenblatt process. ESAIM Probab. Stat., 12:230-257, 2008.

[33] M. S. Veillette and M. S. Taqqu. Berry-Esseen and Edgeworth approximations 
for the tail of an infinite sum of weighted gamma random variables, 2010. To appear in Stochastic Processes and its Applications. Preprint available online at http://arxiv.org/abs/1010.3948.

[34] M. S. Veillette and M. S. Taqqu. A technique for computing the pdfs and cdfs of non-negative infinitely divisible random variables. Journal of Applied Probability 48: 217-237, 2011.

[35] M. S. Veillette and M. S. Taqqu. Supplement to "Properties and numerical evaluation of the Rosenblatt distribution", 2011

[36] A. H. Zemanian. Distribution Theory and Transfor Analysis. Dover, New York, 1987. 